EPJ Web of Conferences 64, 05003 (2014)

DOI: $10.1051 /$ epjconf/ 20146405003

(C) Owned by the authors, published by EDP Sciences, 2014

\title{
3D MHD Simulations of Waves Excited in an Accretion Disk by a Rotating Magnetized Star
}

\author{
R.V.E. Lovelace ${ }^{1, a}$, M.M. Romanova ${ }^{1, b}$ \\ ${ }^{1}$ Department of Astronomy, Cornell University, Ithaca, NY 14853-6801, USA
}

\begin{abstract}
We present results of global 3D MHD simulations of warp and density waves in accretion disks excited by a rotating star with a misaligned dipole magnetic field. A wide range of cases are considered. We find for example that if the star's magnetosphere corotates approximately with the inner disk, then a strong one-arm bending wave or warp forms. The warp corotates with the star and has a maximum amplitude $\left(\left|z_{w}\right| / r \sim 0.3\right)$ between the corotation radius and the radius of the vertical resonance. If the magnetosphere rotates more slowly than the inner disk, then a bending wave is excited at the disk-magnetosphere boundary, but it does not form a large-scale warp. In this case the angular rotation of the disk $[\Omega(r, z=0)]$ has a maximum as a function of $r$ so that there is an inner region where $d \Omega / d r>0$. In this region we observe radially trapped density waves in approximate agreement with the theoretical prediction of a Rossby wave instability in this region.
\end{abstract}

\section{Introduction}

Different types of accreting stars have dynamically important magnetic fields, for example, young $\mathrm{T}$ Tauri stars [1], accreting millisecond pulsars [2], and accreting white dwarfs [3]. Doppler tomography of classical T Tauri stars (CTTSs) shows that in spite of the complexity of the magnetic field at the surface of the star, the dipole component is likely the dominant component at the disk-magnetosphere boundary [4].

A rotating star with a tilted dipole (or other non-axisymmetric) magnetic field exerts a periodic force on the inner part of the accretion disk, which excites different types of waves that propagate to larger distances. This force can excite density waves in the disk [5] and/or vertical bending waves in the disk [6]. These waves can have important observational consequences, such as periodically obscuring the light from the star (e.g., [7]) and periodically modulating the radiation from the inner disk and the star, possibly giving rise to observed quasi-periodic oscillations (QPOs) in low-mass Xray binary systems [2]. Theoretical investigations of small amplitude linearized waves in disks has led to understanding that both types of waves may be strongly enhanced or damped at radii corresponding to resonances (e.g., [8]). There are two main types of linear perturbations. One involves the motion of matter predominantly in the plane of the disk. The other involves the vertical or out of plane motion of the matter. There are important Lindblad resonances associated with the in-plane and out of

\footnotetext{
a e-mail: lovelace@astro.cornell.edu

be-mail: romanova@astro.cornell.edu
}

This is an Open Access article distributed under the terms of the Creative Commons Attribution License 2.0, which permits unrestricted use, distribution, and reproduction in any medium, provided the original work is properly cited. 


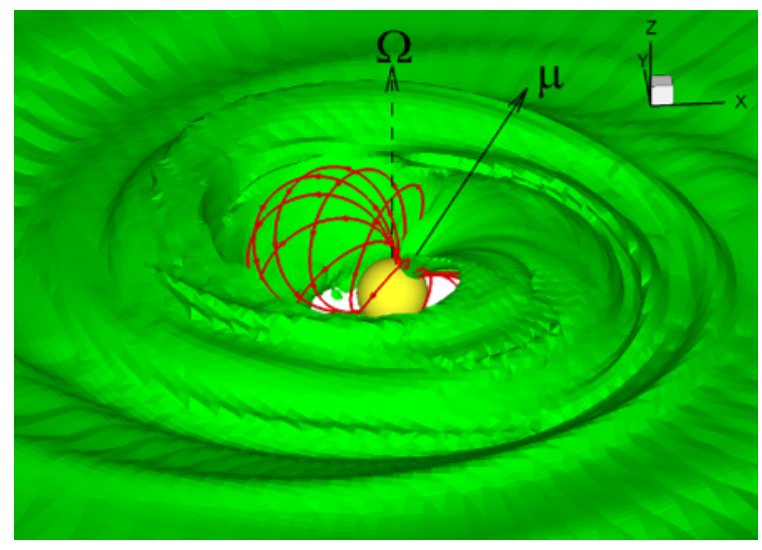

Figure 1. Example of a warp driven by the rotating and tilted at $\theta=30^{\circ}$ dipole magnetosphere for a sample run. The background shows one density level $(\rho=0.07)$. The lines are sample magnetic field lines of the closed magnetosphere. The vectors $\mu$ and $\Omega$ show the directions of the magnetic moment and rotation axis of the star.

plane motion. Another important resonance is associated with corotation radius $r_{\mathrm{cr}}$ where the angular velocity of the disk matches the angular velocity of the perturbation. The linear theory is of course not applicable when the fractional amplitude of the waves is large. In this limit simulations are essential.

Our simulations also give evidence of a Rossby wave instability in the inner region of the disks around accreting magnetized stars where the matter rotation is strongly non-Keplerian because of the stellar magnetic field as proposed by [9]. The theory of the Rossby wave instability (RWI) for Keplerian accretion disks was developed by [10] for thin accretion disks with negligible self-gravity. Earlier, [11] developed a theory of the related instability of thin disk galaxies where the self-gravity may or may not be important and the rotation is not Keplerian. In the first case, the instability can occur if there is a bump as a function of radius in the inverse potential vorticity $F(r) \sim \Sigma[(\nabla \times \mathbf{u}) \cdot \hat{\mathbf{z}}]^{-1}$ at some radius $r_{0}$, where $\Sigma$ is the surface mass density, $\Omega$ the angular velocity, and $\mathbf{u}$ the flow velocity of the disc. For a self-gravitating disk, instability may occur at the radial location of a minimum in $F(r)([11]-[14])$.

Here, we summarize results from the first global 3D magnetohydrodynamic simulations of the waves excited in an accretion disk by a rotating star with a dipole magnetic field misaligned with the star's rotation axis (which is aligned with the disk's rotation axis at large distances) [15]. In Sect. 2 we outline the theory. Sect. 3 gives sample results from our MHD simulations and Sect. 4 discusses the Rossby waves found in our simulations.

Figure 1 shows the driven bending wave or warp excited in a disk by a rotating star with a misaligned magnetic field from [15].

\section{Theory}

The small amplitude waves in a hydrodynamic disk are characterized by the perturbations of the different fluid variables, $\Psi=p, \rho, u_{r}, .$. , which can be expanded as (e.g., [10]): $\Psi(r, z, \phi, t)=$ $\sum_{m, n, \omega} \psi_{m, n, \omega}(r) H_{n}(z / h) \exp (\mathrm{i} m \phi-\mathrm{i} \omega t)$. Here, $\omega$ is the angular frequency of the wave, $m=0,1,2 \ldots$ 

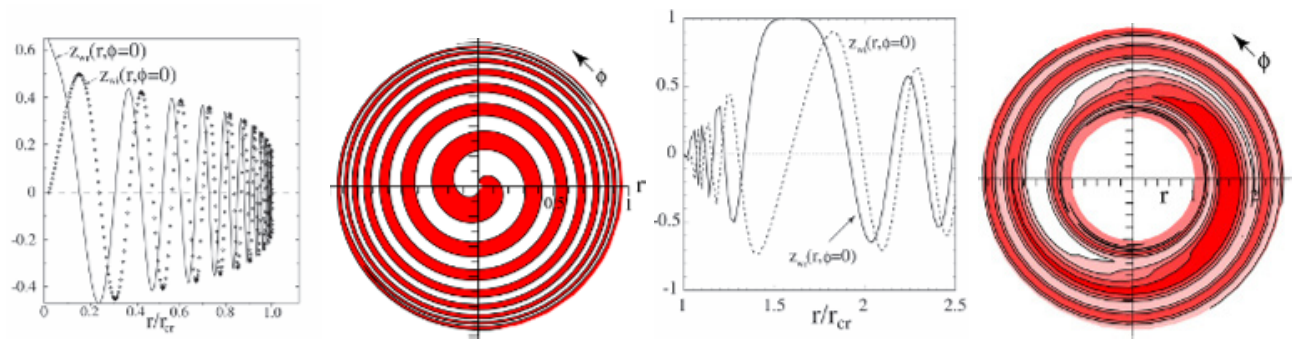

Figure 2. From left to right: (1) Profiles of the real and imaginary parts of the displacement of the disc $z_{w}(r, \phi=0)$ for the 'inner warp', $r / r_{c r}<1$, assuming $c_{s} /(r \Omega)=0.05$. (2) Vertical displacement of the disc $z_{w}(r, \phi)$ for the 'inner warp', $r / r_{\text {cr }}<1$, assuming $c_{s} /(r \Omega)=0.05$. Here, white $=$ negative and dark $=$ positive. (3) Real and imaginary parts of the disc displacement $z_{w}$ for the 'outer warp' from equation A1. (4) Vertical displacement of the disc $z_{w}(r, \phi)$ for the 'outer warp' for $1<r / r_{\mathrm{cr}}<2.5$ assuming $c_{s} /(r \Omega)=0.05$.

is the number or arms in the azimuthal direction, $H_{n}, n=0.1, \ldots$ are Hermite polynomials, and $h(r)$ the half-thickness of the disk at radius $r$. We restrict our attention to the low order modes $m=1,2$ and $n=0,1$.

Linearization of the equations of motion [8] leads to a second order equation of the form $d^{2} \psi / d r^{2}=.$. , where $\psi(r)=\delta p / \rho_{0}$ is the perturbation of the enthalpy. For the bending modes $\psi$ is the displacement of the disk center of mass from the equatorial plane, $z_{w}$. Also in this equation, $\tilde{\omega} \equiv \omega-m \Omega$ is the Doppler-shifted angular frequency of the wave seen by an observer orbiting at the disk's angular velocity $\Omega(r), \Omega_{\perp}$ is the frequency of oscillations perpendicular to the disk, $\kappa^{2}=r^{-3} d\left(r^{4} \Omega^{2}\right) / d r$ is the square of the radial epicyclic frequency, $k_{r}$ is the radial wavenumber, and $c_{s}$ is the sound speed in the disk. Considering the local free wave solution in the form of the WKBJ approximation [8],

$$
\psi \propto k_{r}^{-1 / 2} \exp \left[\mathrm{i} \int^{r} d r k_{r}(r)\right],
$$

for $\left(k_{r} r\right)^{2} \gg 1,\left(k_{r} h\right)^{2} \ll 1$. Thus, $d^{2} \psi / d r^{2}=-k_{r}^{2} \psi$ in equation (1) and one gets the dispersion relation

$$
\frac{\left(\tilde{\omega}^{2}-\kappa^{2}\right)\left(\tilde{\omega}^{2}-n \Omega_{\perp}^{2}\right)}{\tilde{\omega}^{2}}=k_{r}^{2} c_{s}^{2} .
$$

Propagation of the perturbations is possible in regions where $\left(\tilde{\omega}^{2}-\kappa^{2}\right)\left(\tilde{\omega}^{2}-n \Omega_{\perp}^{2}\right)>0$. Part of the range where $\tilde{\omega}^{2}<\kappa^{2}$ describes density waves (inertial-acoustic) waves and part of that with $\tilde{\omega}^{2}>n \Omega_{\perp}^{2}$ describes bending waves. The WKBJ approximation breaks down in the vicinity of points where the coefficient in front of $\psi$ in equation (1) changes sign or has a singularity. At the points where $\tilde{\omega}= \pm \kappa$, there is an outer (+sign) or an inner (-sign) Lindblad resonance. At the points where $\tilde{\omega}= \pm n \Omega_{\perp}$, there are vertical resonances. For $\tilde{\omega}=0$ there is a corotation resonance.

An important class of waves in the disk are the waves "driven" by the distributed force from the rotating tilted magnetosphere of the star. The interaction of the magnetosphere with the disk involves complex, non-linear, and non-stationary processes. The force can be written as $f=f\left(r, z, \phi-\Omega_{*} t\right)=$ $\sum_{m} f_{m}(r, z) \exp \left[\mathrm{i} m\left(\phi-\Omega_{*} t\right)\right]$, where $\Omega_{*}$ is the angular velocity of the star. In linear approximation, we suggest that the $m$-harmonic of this force excites the $m$-arm wave in the disk. The frequency of this force is $m \Omega_{*}$. Then, for a Keplerian disk, the condition for the Lindblad resonances $\tilde{\omega}= \pm \Omega$ is $m \Omega_{*}-m \Omega= \pm \Omega$, or, $r_{\mathrm{LR}}=r_{\mathrm{cr}}(1 \pm 1 / m)^{2 / 3}$, where $r_{\mathrm{cr}}=\left(G M / \Omega_{*}^{2}\right)^{1 / 3}$ is the corotation radius. 

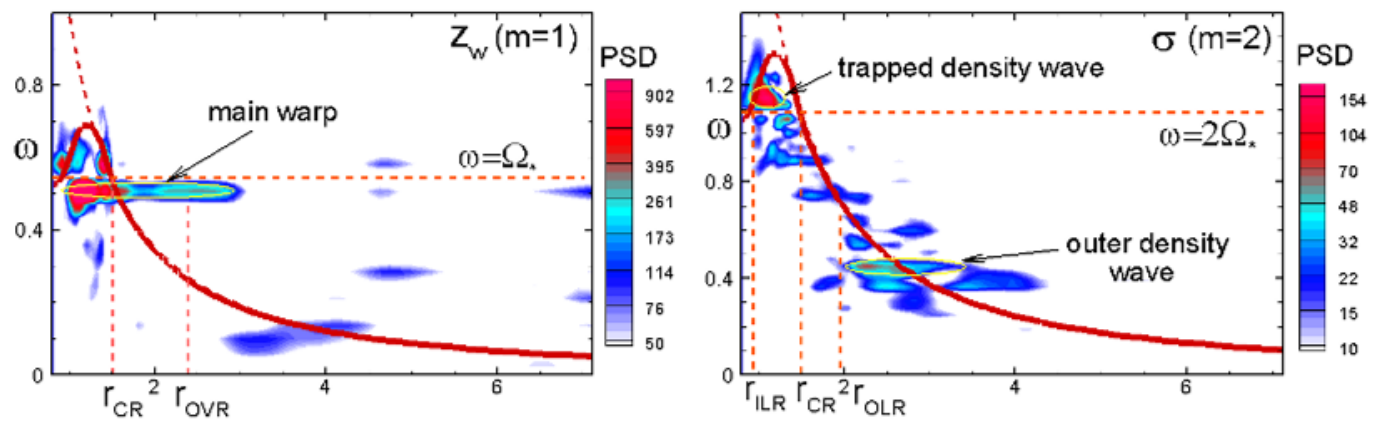

Figure 3. Power spectral densities (PSD) for the $m=1$ warp wave and the $m=2$ in plane density wave $(\sigma)$. The thick solid curves show the average angular velocity $\Omega$ of the disk matter. At small radii the angular velocity decreases owing to the slow rotation rate of the star. This give $d \Omega / d r<0$ at small $r$.

For bending waves, $n=1$, the condition for vertical resonances $\tilde{\omega}= \pm \Omega$ is the same, and hence the vertical resonances are located at the same radii.

Figure 2 shows sample results for the bending or warp waves predicted by the WKBJ theory [15].

\section{3D MHD Simulations}

We solve the 3D MHD equations with a Godunov-type code in a reference frame rotating with the star using the "cubed sphere" grid. The model has been described in detail earlier [17]. Hence, we describe it only briefly here. For initial conditions we assume that the rotating, perfectly conducting, magnetized star is surrounded by an accretion disk and a corona. The disk is cold and dense, while the corona is hot and rarefied, and at the reference point (the inner edge of the disk in the disk plane), $T_{c}=100 T_{d}$, and $\rho_{c}=0.01 \rho_{d}$, where the subscripts 'd' and 'c' denote the disk and the corona. The disk and corona are initially in rotational hydrodynamic equilibrium. The disk is relatively thin, with the half-thickness to radius ratio $h / r \approx 0.1$. The grid consists of $N_{r}$ concentric spheres, where each sphere represents an inflated cube. Each of the six sides of the inflated cube has an $N \times N$ curvilinear Cartesian grid. The whole grid consists of $6 \times N_{r} \times N^{2}$ cells. The typical grid used in simulations has $N^{2}=61^{2}$ angular cells in each block. The typical number of grid cells in the radial direction is $N_{r}=140$.

Figure 3 shows the power spectral densities (PSD) for the $m=1$ warp wave $\left(z_{w}\right)$ and the $m=2$ density wave $(\sigma)$ from [15]. The left-hand panel shows that the warp wave rotates at the angular frequency of the star and that it extends radially outward and inward from the corotation radius. It is in approximate agreement with the theory of [19]. The right-hand panel shows the PSD of the density waves. Note, in particular, the prominent feature on the inner part of the rotation curve where $d \Omega / d r<0$. We identify this feature as a Rossby wave as discussed below.

\section{Radially Trapped Rossby Modes}

Of particular interest here is the Rossby wave instability in the non-Keplerian region of a disk where $d \Omega / d r>0$ predicted theoretically by [9]. This region occurs when a disk encounters the magnetosphere of a slowly rotating star. For given profiles of $v_{\phi}, \rho$, etc. and a given $m$, the value of the growth 


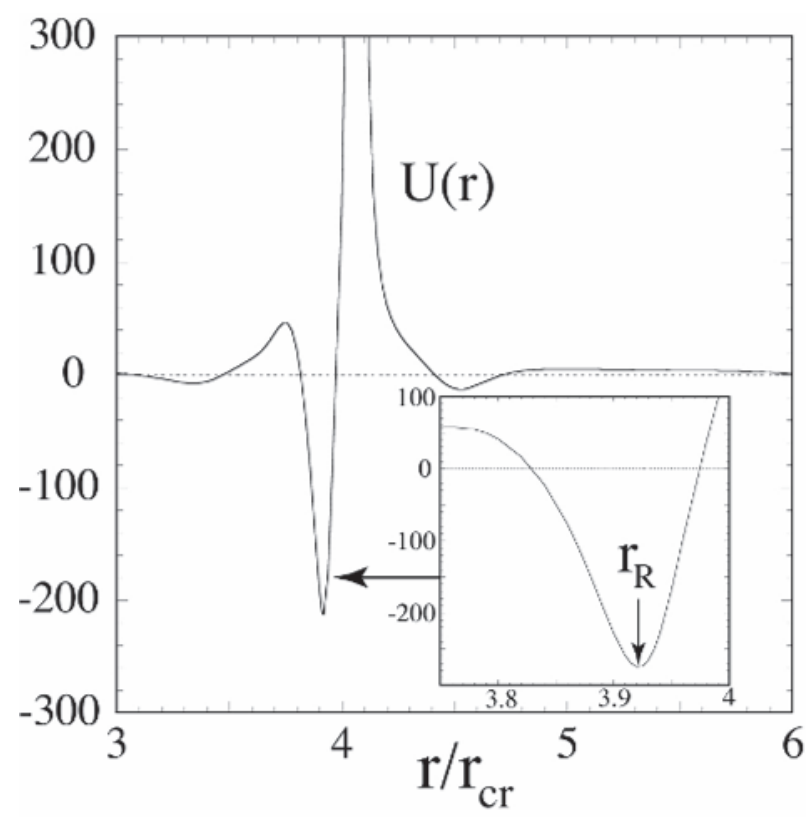

Figure 4. The effective potential for the Rossby waves in the inner region of the disk where $d \Omega / d r>0$ for $m=1$ [9] The vertical arrow at $r_{R}$ indicates the bottom of the potential well.

rate is found by solving for the 'ground state' solution of Schrödinger-like equation for the enthalpy perturbation $\psi=\delta p / \rho$,

$$
\frac{d^{2} \psi}{d r^{2}}=U(r \mid \omega) \psi
$$

where $U(r \mid \omega)$ is an effective potential shown for a sample case in Figure 4 from [9]. The ground state is found by varying both the real and imaginary parts of the mode frequency $\omega$ so as to give the $\psi(r)$ most deeply trapped in the potential well. For typical profiles and $m=1$, the growth rate is $\omega_{i} \sim 0.1\left(v_{\phi} / r\right)_{R}$ and the real part of the frequency is $\omega_{r} \approx\left(v_{\phi} / r\right)_{R}$, where the $R$-subscript indicates evaluation at the resonant radius $r_{R}$. The radial width of the mode is $\Delta r / r_{R} \sim 0.05$.

The linear growth of the Rossby wave is predicted to saturate when the azimuthal frequency of trapping of a fluid particle in the trough of the wave $\omega_{T}$ grows to a value equal to the growth rate $\omega_{i}$ as discussed in [9]. Recently, the saturation of the Rossby wave growth has been studied in a large set of simulations of bumps in $F(r)$ on Keplerian disks and the saturation in most cases is found to correspond to $\omega_{T} \approx \omega_{i}[20]$.

\section{Acknowledgments}

We thank the conference organizers, particularly E. Bozzo for organizing an excellent conference. Further, we thank G. V. Ustyugova and A. V. Koldoba for the development of the codes used in the reported simulations. Resources supporting this work were provided by the NASA High-End Computing (HEC) Program through the NASA Advanced Supercomputing (NAS) Division at Ames Research Center and the NASA Center for Computational Sciences (NCCS) at Goddard Space Flight 
Center. The research was supported by NASA grant NNX11AF33G and NSF grants AST-1008636 and AST-1211318.

\section{References}

[1] Bouvier, J., Alencar, S. H. P., Harries, T. J., Johns-Krull, C. M. \& Romanova, M. M. 2007, in Protostars and Planets V, B. Reipurth, D. Jewitt, and K. Keil (eds.), University of Arizona Press, Tucson, 479

[2] van der Klis, M. 2006, in Compact Stellar X-Ray Sources, Eds. W.H.G. Lewin \& M. van der Klis (Cambridge: Cambridge Univ. Press), p. 39

[3] Warner, B. 2004, PASP, Volume 116, Issue 816, pp.115, Rapid Oscillations in Cataclysmic Variables

[4] Donati, J.-F., Skelly, M. B., Bouvier, J., Gregory, S. G., Grankin, K. N., Jardine, M. M., Hussain, G. A. J., Ménard, F., Dougados, C., Unruh, Y., Mohanty, S., Aurièfre, M., Morin, J. \& Farès, R. 2010, MNRAS, 409, 1347

[5] Goldreich, P., \& Tremaine, S. 1978, ApJ, 222, 850

[6] Lubow, S.H. 1981, ApJ, 245, 274

[7] Bouvier, J., Alencar, S. H. P., Boutelier, T., Dougados, C., Balog, Z., Grankin, K., Hodgkin, S. T., Ibrahimov, M. A., Kun, M., Magakian, T. Yu., Pinte, C. 2003, A\&A, 463, 1017

[8] Kato, S., Fukue, J., \& Mineshige, S. 1998, Black-Hole Accretion Disks (Kyoto University Press: Kyoto Japan)

[9] Lovelace, R. V. E., Turner, L. \& Romanova, M. M. 2009, ApJ, 701, 225

[10] Lovelace, R.V.E., Li, H., Colgate, S.A., \& Nelson, A.F. 1999, ApJ, 513, 805

[11] Lovelace, R.V.E., \& Hohlfeld, R.G. 1978, ApJ, 221, 51

[12] Lyra, W., Johansen, A., Klahr, H., \& Piskunov, N. 2008, A\&A, 491, L41

[13] Lin, M.-K., \& Papaloizou, J. C. B. 2011, MNRAS, 415, 1426

[14] Lovelace, R.V.E., \& Hohlfeld, R.G. 2013, MNRAS, 429, 529

[15] Romanova, M.M., Ustyugova, G.V., Koldoba, A.V., \& Lovelace, R.V.E. 2013, MNRAS, 430, 699

[16] Lai, D. \& Zhang, H. 2008, ApJ, 683, 949

[17] Koldoba, A. V., Romanova, M. M., Ustyugova, G. V., Lovelace, R. V. E. 2002, ApJ, 576, L53

[18] Romanova, M.M., Ustyugova, G.V., Koldoba, A.V., \& Lovelace, R.V.E. 2002, ApJ, 578, 420

[19] Terquem, C., \& Papaloizou, J.C.B. 2000, A\&A, 360, 1031

[20] Meheut, H., Lovelace, R.V.E., \& Lai, D. 2013, MNRAS, 430, 1988 